\title{
A Two-Phase Free Boundary Problem for the Nonlinear Heat Equation
}

\author{
$S D E L I L L O^{\dagger}$ and $M C S A L V A T O R I^{\ddagger}$ \\ † Dipartimento di Matematica e Informatica, Università di Perugia \\ Via Vanvitelli, 1, Perugia, Italy \\ ¥ Istituto Nazionale di Fisica Nucleare, Sezione di Perugia, Italy
}

Received June 18, 2003; Accepted September 09, 2003

\begin{abstract}
A two-phase free boundary problem associated with nonlinear heat conduction is considered. The problem is mapped into two one-phase moving boundary problems for the linear heat equation, connected through a constraint on the relative motion of their moving boundaries. Existence and uniqueness of the solution is proved for small times and a particular exact solution is discussed.
\end{abstract}

Free Boundary Problems (FBP) motivated several studies in the past due to their relevance in applications $[1-4]$. From the mathematical point of view FBP are initial/ boundary value problems with a moving boundary [5]. The motion of the boundary is unknown (free boundary) and has to be determined together with the solution of the given partial differential equation. As a consequence the solution of FBP is in most cases equivalent to the solution of a nonlinear system. In recent studies $[6-13]$ some free boundary problems for nonlinear evolution equations relevant in applications have been considered. In particular in [12] the solution of a one-phase Stefan Problem in nonlinear conduction is proved to exist and to be unique for short intervals of time. Furthemore a particular travelling wave solution was obtained. On the other hand two-phase Stefan Problems are more complicated than their one-phase counterparts and the theory is more elaborate.

It is the aim of this paper to analyse a two-phase Stefan Problem for the nonlinear heat equation considered in [12].

Such an equation arises as a model of heat conduction in solid crystalline hydrogen [14]. It admits an exact linearization into the heat equation and therefore belongs to the class of C-integrable equations [16].

In the following we show that the two-phase Stefan Problem for the nonlinear heat equation admits a linearization into two distinct one-phase moving boundary problems for the linear heat equation. The two linearized problems are connected through a constraint on the relative motion of their moving boundaries. Such a constraint is induced by the free boundary motion of the nonlinear problem.

We start our analysis with the following system of nonlinear heat equations

$$
\frac{\vartheta_{1 t}}{\vartheta_{1}^{2}}=\delta_{1} \vartheta_{1 x x}, \quad \vartheta_{1}=\vartheta(x, t),
$$


over the domain $x \in(-\infty, s(t)), t>0$, and

$$
\frac{\vartheta_{2 t}}{\vartheta_{2}^{2}}=\delta_{2} \vartheta_{2 x x}, \quad \vartheta_{2}=\vartheta_{2}(x, t)
$$

over the domain $x \in(s(t),+\infty), t>0$, where

$$
s(0)=b>0 .
$$

Equations (1) and (2) have initial data given by

$$
\begin{array}{ll}
\theta_{1}(x, 0)=\varphi_{1}(x), & x \in(-\infty, b), \\
\theta_{2}(x, 0)=\varphi_{2}(x), & x \in(b,+\infty),
\end{array}
$$

related through a continuity condition at the free boundary

$$
\varphi_{1}(b)=\varphi_{2}(b)=\alpha<0 .
$$

Moreover (1) and (2) are characterized by the following set of boundary conditions

$$
\vartheta_{1}(-\infty, t)=\beta_{1}>0, \quad \vartheta_{2}(+\infty, t)=\beta_{2}<0,
$$

where $t \geqslant 0$ and

$$
\vartheta_{1}(s(t), t)=\vartheta_{2}(s(t), t)=\alpha .
$$

Equation (7) together with a condition on the heat flux [see (8) below] is sufficient to determine the motion of the free boundary $s(t)$. In the above relations $\delta_{j}(j=1,2)$ are positive constants related to the thermal conductivity of the two phases; $\beta_{1}, \beta_{2}$ and $\alpha$ are constants $\left(|\alpha|<\left|\beta_{2}\right|\right)$ and the unknown function $s(t)$ describes the motion of the free boundary and has to be determined together with $\vartheta_{j}(x, t)(j=1,2)$. Besides $(7)$ the system is characterized by an additional condition at the free boundary, $s(t)$, stemming from thermal energy considerations. The energy balance across the free boundary can be written as

$$
-k_{1} \vartheta_{1 x}(s(t), t)+k_{2} \vartheta_{2 x}(s(t), t)=\frac{d s}{d t},
$$

where $k_{j}=\delta_{j} / \lambda(j=1,2)$, with $\lambda$ denoting the latent heat of fusion.

In order to linearize (1) and (2) we introduce the transformations

$$
\psi_{j}(z, t)=\vartheta_{j}(x, t), \quad j=1,2, \quad z=z(x, t),
$$

with

$$
\begin{aligned}
& z_{x}=\frac{1}{\vartheta_{1}}, \quad z_{t}=-\delta_{1} \vartheta_{1 x}, \quad x \in(-\infty, s(t)), \\
& z_{x}=\frac{1}{\vartheta_{2}}, \quad z_{t}=-\delta_{2} \vartheta_{2 x}, \quad x \in(s(t),+\infty),
\end{aligned}
$$


the compatibility of which, $z_{x t}=z_{t z}$, is easily proved by (1) and (2). Under the above transformations we obtain from (1) and (2) the following system of linear heat equations

$$
\begin{array}{ll}
\psi_{1 t}=\delta_{1} \psi_{1 z z}, & -\infty<z<\bar{z}_{1}(t), \\
\psi_{2 t}=\delta_{2} \psi_{2 z z}, & \bar{z}_{2}(t)<z<+\infty,
\end{array}
$$

where

$$
\bar{z}_{1}(t)=\lim _{x \nearrow s(t)} z(x, t), \quad \bar{z}_{2}(t)=\lim _{x \searrow s(t)} z(x, t) .
$$

It is worth noting that the two limits in relation (14) are different. In fact, when (9) and (10) are used together with the flux boundary condition (8), we get

$$
\frac{d \bar{z}_{1}}{d t}-\frac{d \bar{z}_{2}}{d t}=\lambda \frac{d s}{d t}
$$

which can be integrated giving

$$
\bar{z}_{1}(t)-\bar{z}_{2}(t)=\lambda[s(t)-b] .
$$

The above relations imply that equations (12) and (13) are defined over semi-infinite domains with distinct moving boundaries, given by (14). Relation (16) shows that the two moving boundaries, $\bar{z}_{1}(t)$ and $\bar{z}_{2}(t)$, are forced into a relative motion induced by the motion of the free boundary of the nonlinear problem. The relative velocity of the two boundaries is given by (15) and is proportional to the velocity $d s / d t$ of the free boundary motion. The linear equations (12) and (13) are characterized by the set of initial data

$$
\psi_{j}\left(z_{0}, 0\right)=\varphi_{j}(x), \quad j=1,2, \quad z_{0} \equiv z(x, 0),
$$

and by the boundary conditions

$$
\psi_{1}(-\infty, t)=\beta_{1}, \quad \psi_{2}(+\infty, t)=\beta_{2} .
$$

Together with the boundary conditions at the moving boundaries

$$
\psi_{1}\left(\bar{z}_{1}(t), t\right)=\psi_{2}\left(\bar{z}_{2}(t), t\right)=\alpha
$$

and

$$
-k_{1} \psi_{1 z}\left(\bar{z}_{1}(t), t\right)+k_{2} \psi_{2 z}\left(\bar{z}_{2}(t), t\right)=\alpha \frac{d s}{d t},
$$

where (4), (5), (7) and (8) have been used.

The two-phase nonlinear Stefan Problem (1) and (2) has then been mapped into two distinct moving boundary problems for the linear heat equations (12) and (13) with initial data (17) and boundary conditions (18)-(20). The two problems are not independent from each other. They are connected via (15) and (16) which put a constraint on the relative motion of the boundaries. There is moreover an additional constraint on the heat flux across the moving boundaries given by (20). Such constraints are both induced by the motion of the free boundary in the two-phase Stefan Problem. 
We say that $\left\{\psi_{j}(z, t), \bar{z}_{j}(t),(j=1,2)\right\}$ form a solution of the above moving boundary problems for all $t<\sigma, \quad 0<\sigma<\infty$, when : (a) $\psi_{j}(z, t)(j=1,2)$ are solutions of $(12)$, (13) satisfying (17)-(20), they exist and are continuous together with their derivatives; (b) $\bar{z}_{j}(t)(j=1,2)$ are continuously differentiable functions on $[0, \sigma)$.

In order to prove the existence and uniqueness of the solution for $t<\sigma$, we assume that the initial data $\varphi_{j}(j=1,2)$ given in (4) and (5) are continuous together with their derivatives. Moreover they are bounded:

$$
\left|\varphi_{1}(x)\right|<\beta_{1}, \quad\left|\varphi_{2}(x)\right|<\left|\beta_{2}\right|
$$

with $\beta_{1}$ and $\beta_{2}$ given by (6).

Next we turn our attention to the solution of (12) and (13). To this end we first recall the fundamental kernel of the heat equation

$$
K_{j}\left(z-\xi, t-t^{\prime}\right)=\frac{1}{2 \sqrt{\pi \delta_{j}}} \frac{1}{\sqrt{t-t^{\prime}}} \exp \left(-\frac{(z-\xi)^{2}}{4 \delta_{j}\left(t-t^{\prime}\right)}\right), \quad j=1,2,
$$

and the Green's identity for the heat equation, namely

$$
\frac{\partial}{\partial \xi}\left(K_{j} \frac{\partial \psi_{j}}{\partial \xi}-\psi_{j} \frac{\partial K_{j}}{\partial \xi}\right)=\frac{\partial}{\partial t}\left(K_{j} \psi_{j}\right), \quad j=1,2
$$

Integrating (23) on the domain $-\infty<\xi<\bar{z}_{j}\left(t^{\prime}\right)$ when $j=1 \quad\left[\bar{z}_{2}\left(t^{\prime}\right)<\xi<\infty\right.$ when $j=2], \quad 0<\varepsilon<t^{\prime}<t-\epsilon$, and letting $\varepsilon \rightarrow 0$, since $\psi_{j}\left(\bar{z}_{j}(t), t\right)=\alpha$ and $K_{j}(z-\xi, 0)=$ $\delta(z-\xi)$, we obtain

$$
\begin{gathered}
\psi_{1}(z, t)=\int_{-\infty}^{\bar{b}_{1}} K_{1}(z-\xi, t) \varphi_{1}(\xi) d \xi-\frac{1}{\alpha} \int_{0}^{t} K_{1}\left(z-\bar{z}_{1}\left(t^{\prime}\right), t-t^{\prime}\right) \psi_{1 z}\left(\bar{z}_{1}\left(t^{\prime}\right), t^{\prime}\right) d t^{\prime} \\
-\alpha \int_{0}^{t} K_{1 \xi}\left(\bar{z}_{1}\left(t^{\prime}\right), t-t^{\prime}\right) d t^{\prime}, \\
\begin{array}{r}
\psi_{2}(z, t)=\int_{\bar{b}_{2}}^{\infty} K_{2}(z-\xi, t) \varphi_{2}(\xi) d \xi+\frac{1}{\alpha} \int_{0}^{t} K_{2}\left(z-\bar{z}_{2}\left(t^{\prime}\right), t-t^{\prime}\right) \psi_{2 z}\left(\bar{z}_{1}\left(t^{\prime}\right), t^{\prime}\right) d t^{\prime} \\
+\alpha \int_{0}^{t} K_{2 \xi}\left(\bar{z}_{2}\left(t^{\prime}\right), t-t^{\prime}\right) d t^{\prime},
\end{array}
\end{gathered}
$$

where, due to (4.b) and (4.c), we have

$$
\begin{aligned}
& \bar{z}_{j}(t)=h_{j}(t)-\frac{\delta_{j}}{\alpha} \int_{0}^{t} \psi_{j z}\left(\bar{z}_{j}\left(t^{\prime}\right), t^{\prime}\right) d t^{\prime}, \quad j=1,2, \\
& h_{1}(t)=\int_{x_{1} 0}^{s(t)} \frac{d x^{\prime}}{\varphi_{1}\left(x^{\prime}\right)}, \quad h_{2}(t)=\int_{s(t)}^{x_{2}} \frac{d x^{\prime}}{\varphi_{2}\left(x^{\prime}\right)}
\end{aligned}
$$

and $\bar{b}_{j} \equiv h_{j}(0), \quad j=1,2$. 
In the right hand side of $(24)[(25)] \psi_{1 z}\left(\bar{z}_{1}(t), t\right)\left[\left(\psi_{2 z}\left(\bar{z}_{2}(t), t\right)\right]\right.$ is unknown. It is convenient to take the $x$-derivative of both sides in (24) [(25)] and take its limit as $z \nearrow \bar{z}_{1}(t)$ $\left[z \backslash \bar{z}_{2}(t)\right]$. We then put $w_{j}(t)=\psi_{j z}\left(\bar{z}_{j}(t), t\right), \quad j=1,2$, and finally obtain [13]

$$
\begin{aligned}
w_{1}(t)= & \left(1+\frac{1}{2 \alpha}\right)^{-1}\left[-\varphi_{1}\left(\bar{b}_{1}\right) K_{1}\left(\bar{z}_{1}(t)-\bar{b}_{1}, t\right)+\int_{-\infty}^{\bar{b}_{1}} K_{1}\left(\bar{z}_{1}(t)-\xi, t\right) \varphi_{1}^{\prime}(\xi)\right. \\
& \left.-\frac{1}{\alpha} \int_{0}^{t} K_{1 z}\left(\bar{z}_{1}(t)-\bar{z}_{1}\left(t^{\prime}\right), t-t^{\prime}\right) w_{1}\left(t^{\prime}\right) d t^{\prime}-\alpha \int_{0}^{t} K_{1 t^{\prime}}\left(\bar{z}_{1}\left(t^{\prime}\right), t-t^{\prime}\right) d t^{\prime}\right] \\
w_{2}(t)= & \left(1+\frac{1}{2 \alpha}\right)^{-1}\left[-\varphi_{2}\left(\bar{b}_{2}\right) K_{2}\left(\bar{z}_{2}(t)-\bar{b}_{2}, t\right)+\int_{\bar{b}_{2}}^{\infty} K_{2}\left(\bar{z}_{2}(t)-\xi, t\right) \varphi_{2}^{\prime}(\xi)\right. \\
& \left.-\frac{1}{\alpha} \int_{0}^{t} K_{2 z}\left(\bar{z}_{2}(t)-\bar{z}_{2}\left(t^{\prime}\right), t-t^{\prime}\right) w_{2}\left(t^{\prime}\right) d t^{\prime}-\alpha \int_{0}^{t} K_{2 t^{\prime}}\left(\bar{z}_{2}\left(t^{\prime}\right), t-t^{\prime}\right) d t^{\prime}\right]
\end{aligned}
$$

with

$$
\bar{z}_{j}(t)=h_{j}(t)-\frac{\delta_{j}}{\alpha} \int_{0}^{t} w_{j}\left(t^{\prime}\right) d t^{\prime}, \quad j=1,2 .
$$

Thus the solution of the free boundary problems (15), (16), (17)-(20) has been reduced to the solution of the system of nonlinear integral equations (28)-(30).

Once the existence and uniqueness of the functions $w_{j}(t)(j=1,2)$ is proved for $0 \leqslant$ $t<\sigma$, the existence and uniqueness of $\varphi_{j}(x, t)(j=1,2)$ then follows via $(24)$ and $(25)$. The solution of the two-phase Stefan Problem (1)-(8) for the nonlinear heat equation then exists and is unique (for $0 \leqslant t<\sigma$ ) due to (9)-(11), with $s(t)$ given by (16). Equations (28), (29) and (30) are of the same type as equations (4) and (5) of [12]. Existence and uniqueness of the solutions $w_{j}(t)(j=1,2)$ for small times can therefore be established through the same analysis reported in Section 3 of [12], which is not repeated here.

We now turn our attention to a particular solution of the two-phase Stefan Problem (1)-(8). Namely we consider a moving front solution of equation (12)

$$
\psi_{1}(z, t)=\beta_{1}\left\{1-\exp \left[-v_{1}\left(z-v_{1} t\right)\right]\right\}, \quad v_{1}<0,
$$

which is travelling to the left with constant speed $v_{1}$ and is compatible with the boundary condition (18). The corresponding solution of (13), compatible with (18), is

$$
\psi_{2}(z, t)=\beta_{2}\left\{1-\exp \left[-v_{2}\left(z-v_{2} t\right)\right]\right\}, \quad v_{2}>0,
$$

and is travelling to the right with constant speed $v_{2}$. We use $(31)[(32)]$ on the interval $-\infty<z<\bar{z}_{1}(t)\left[\bar{z}_{2}(t)<z<\infty\right]$ and require

$$
\psi_{1}(z, t)=0, \quad z>\bar{z}_{1}(t) \quad\left[\psi_{2}(z, t)=0, \quad z<\bar{z}_{2}(t)\right] .
$$

We now impose on $\psi_{1}(z, t)$ and $\psi_{2}(z, t)$ the boundary condition (19). We get

$$
\bar{z}_{1}(t)=\bar{b}_{1}+v_{1} t, \quad \bar{z}_{2}(t)=\bar{b}_{2}+v_{2} t
$$


where the constants $\bar{b}_{j}, j=1,2$, are assumed to be positive. Moreover

$$
\begin{aligned}
& \frac{d \bar{z}_{1}}{d t}=v_{1}=-\frac{1}{\bar{b}_{1}} \log \left(1+\frac{|\alpha|}{\beta_{1}}\right), \\
& \frac{d \bar{z}_{2}}{d t}=v_{2}=-\frac{1}{\bar{b}_{2}} \log \left(1-\left|\frac{\alpha}{\beta_{2}}\right|\right) .
\end{aligned}
$$

The boundary $\bar{z}_{1}(t)\left[\bar{z}_{2}(t)\right]$ and the front solution (31) [(32)] are both moving to the left [right] with the same constant speed. Next, we use (15) and obtain

$$
\frac{d s}{d t}=\lambda^{-1}\left(v_{1}-v_{2}\right)<0
$$

which implies that the free boundary of the Stefan Problem (1)-(8) is moving to the left with constant speed. We impose on $\psi_{1}(z, t)$ and $\psi_{2}(z, t)$ the boundary condition (20), obtaining the following constraints on the parameters $\beta_{1}, \beta_{2}$ and $\alpha$ characterizing the solution (31) and (32)

$$
\frac{\beta_{1}}{|\alpha|}=\frac{1}{\delta_{1}}-1, \quad\left|\frac{\beta_{2}}{\alpha}\right|=\frac{1}{\delta_{2}}-1,
$$

where (36) has also been used.

Finally the solution of the two-phase Stefan Problem for the nonlinear heat equation is given by

$$
\vartheta_{j}(x, t)=z_{x}^{-1}, \quad j=1,2,
$$

where, due to (10) and (11), $z(x, t)$ satisfies

$$
x=\int_{0}^{z} \psi_{j}\left(z^{\prime}, t\right) d z^{\prime} \quad j=1,2
$$

with $\psi_{1}(z, t)\left[\psi_{2}(z, t)\right]$ given by $(31)[(32)]$ and the speed $v_{1}\left(v_{2}\right)$ specified by $(34)[(35)]$.

It is worth noting that the above solution is a particular solution of the Stefan Problem (1)-(8). In fact it corresponds to the special case when the nonlinear integral equations (28) and (29) reduce to linear integral equations of Volterra type in $t$, as implied by substituting (33) back into (28) and (29).

\section{References}

[1] Friedman A, Variational Principles and Free-Boundary Problems, Wiley-Interscience Publication, Wiley, New York, 1982.

[2] Elliott C M and Ockendon J R, Weak and Variational Methods for Moving Boundary Problems, Research Notes in Mathematics vol 59, New York, Pitman, 1982.

[3] Crank J, Free and Moving Boundary Problems, Science Publications, Oxford, 1984.

[4] Friedman A, Not. AMS 47 (2000), 854. 
[5] Fokas A S and Pelloni B, Phys. Rev. Lett. 84 (2000), 4785.

[6] Rogers C, J. Phys. A 18 (1985), L105.

[7] Rogers C, J. Nonlinear Mech. 21 (1986), 249.

[8] Natale M F and Tarzia D A, J. Phys. A: Math. Gen. 33 (2000), 395.

[9] Ablowitz M J and De Lillo S, Nonlinearity 13 (2000), 471.

[10] Ablowitz M J and De Lillo S, Phys. Lett. A 271 (2000), 273.

[11] Ablowitz M J and De Lillo S, J. Phys. A: Math. Gen. 35 (2003), 1.

[12] De Lillo S and Salvatori M C, J. Nonlinear Math. Phys. 9 (2002), 446.

[13] De Lillo S, Salvatori M C and Sanchini G, Phys. Lett. A 310 (2003), 25.

[14] Rosen G, Phys. Rev. B 19 (1979), 2398.

[15] Rosen G, Phys. Rev. B 23 (1981), 3093.

[16] Calogero F, J. Math. Phys. 34 (1993), 3197.

[17] Friedman A, J. Math. Mech. 8 (1959), 499. 